\title{
Blind Identification and Digital Calibration of Volterra Model Based on Least Mean Square Method
}

\author{
Peng Liang, Haihua Deng, Ming Chen \\ Wuhan Second Ship Design and Research Institute \\ Wuhan, Hubei, P.R. China, 430064 \\ E-mail: 284851356@qq.com
}

\begin{abstract}
Nonlinear distortions and memory effect of broadband receiver's front-end are canceled out simultaneously using a digital post-calibration technique based on Volterra model. This paper develops a least mean squared blind identification criterion for the measurement of the model parameters without prior knowledge of the received signals, and the optimization goal could be described as for the minimizing of total energy of the calibrated outputs that located in the first Nyquist band other than strong signals. Frequency locations of the distortions are availably determined by the comparison between the input and output spectrums of a digital nonlinear polynomial with fixed coefficients. Experimental results on the actual nonlinear circuit show that with the proposed technique, $15 \mathrm{~dB}$ improvement in SpursFree-Dynamic-Range with multi-tone excitation signal is achieved. High-speed, high-precision Software Defined Radio systems would benefit much from the novel technical solution presented in the paper.
\end{abstract}

Keywords-Volterra model; Least Mean Square (LMS); memory nonlinearity; blind sysmtem identification

\section{INTRODUCTION}

The widely used Volterra model is an exact mathematical approach for description of the causal time-invariant systems [1] either in time domain or in frequency domain [2]. Several other parametric models, such as the memory polynomial, Hammerstein and Wiener models, can be considered as the simplified format of the Volterra model.

The inverse Volterra model of the memory nonlinearities could be used to post-compensate the unknown spurs for improving the linearization performance of the receiver's front end [3]-[5]. Performance improvement of $5.3 \mathrm{~dB}$ in terms of signal-to-noise ratio was achieved by a-priori approach [3]. Genetic Algorithm was applied to solve a system of nonlinear equations which the kernels of a discrete time Volterra series were the unknown parameters [4]. With the help of extra circuits or components, the distortion target was acquired [5]. In this paper, a blind identification method based on the LMS algorithm is proposed to achieve the inverse Volterra model of the memory nonlinearities for the receiver's front end. This inverse is used to post-compensate the unknown distortion in real-time.

This paper is organized as follows. Section II describes the blind identification method of the Volterra model. The coefficients of the nonlinear compensator are obtained in
Section III. Section IV shows the experimental results. Finally, the conclusions are drawn in Section V.

\section{BLIND IDENTIFICATION OF VOLTERRA MODEL}

The Volterra models make use of functions and kernels to represent a wide class of nonlinear systems with memory where the nonlinearities are small compared to the linear term. For using digital techniques, the symmetric discretetime Volterra model can be written as:

$$
s(k)=y(k)-\sum_{d=2}^{D}\left(\sum_{r_{1}=0}^{N_{d}-1} \ldots \sum_{r_{d}=r_{d-1}}^{N_{d}-1} h\left(r_{1}, r_{2}, \ldots, r_{d}\right) \prod_{j=1}^{d} y\left(k-r_{j}\right)\right)
$$

Where, $y(k)$ is the $k$ th discrete sample of nonlinear system, $s(k)$ is the $k$ th discrete compensated output, $d$ is the nonlinear order, $D$ is the maximum value of $d, N_{d}$ is the memory depth of the model, $h\left(r_{1}, r_{2}, \ldots, r_{d}\right)$ is the Volterra kernel of the $d$ th order.

Number of the nonlinear kernels equals to:

$$
N=\sum_{d=2}^{D} \frac{\left(N_{d}+d-1\right) !}{\left(N_{d}-1\right) ! d !}
$$

As shown in Fig.1, the nonideal characteristics of the receiver's front-end can be evaluated by introducing a cascade of a continuous-time nonlinear dynamic system and an ideal quantization device. Post-compensation is mainly aimed at searching for an inverse of the nonlinear system that would exactly counteract the nonlinearity of the receiver's front-end.

The objective of the blind nonlinear compensation is to minimize the distortion energy at the $M$-sample nonlinear compensator output $s(k)$. The frequency content of $s(k)$ is obtained by employing a $M$-point discrete cosine transform (DCT). Only the signals below the preset threshold which locate in the first Nyquist band are extracted out by means of a bandstop filter.

The total distortion energy $J(\omega)$ can be written as: 


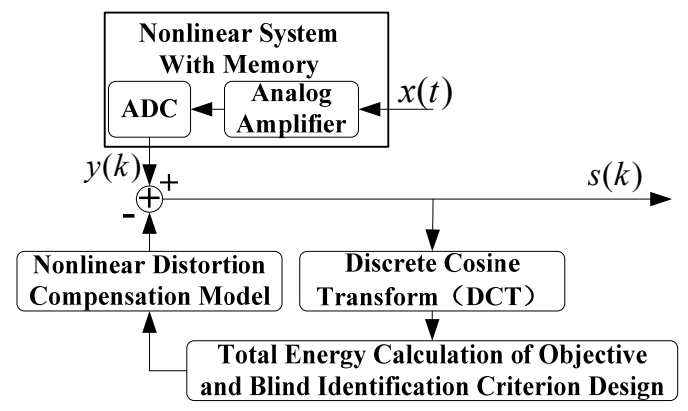

Figure 1. Memory nonlinear system and its post-compensation

$$
\begin{aligned}
& J(\omega)=\left[S_{f}(\omega)\right]^{T} S_{f}(\omega)=\sum_{k=1}^{K}\left[s_{f}(k, \omega)\right]^{2}=\sum_{k=1}^{K}\left[G^{T} S(k)\right]^{2}(3) \\
& \omega=\left[h(0,0) \ldots h\left(N_{2}-1, N_{2}-1\right) \ldots h(0,0,1) \ldots h\left(N_{D}-1, \ldots, N_{D}-1\right)\right]^{T} \\
& S_{f}(\omega)=\left[\begin{array}{llll}
s_{f}(1, \omega) & \left.s_{f}(2, \omega) \quad \ldots \quad s_{f}(K, \omega)\right]^{T}
\end{array}\right. \\
& S(k)=\left[\begin{array}{llllll}
s(k) & s(k-1) & s(k-2) & s(k-3) & \ldots & s(k-B+1)
\end{array}\right]^{T}
\end{aligned}
$$

Where, $\omega$ is the $N \times 1$ Volterra filter's coefficient vector, which is the nonlinear compensator coefficient vector too, $S_{f}(\omega)$ is the $K \times 1$ distortion signal serial vector, $s_{f}(k, \omega)$ is the $k$ th distortion signal, $G=\left[\begin{array}{ll}G_{0} & G_{1} \ldots G_{B-1}\end{array}\right]^{T}$ is the $B \times 1$ coefficient vector of the FIR bandstop filter, $S(k)$ is the $B \times 1$ nonlinear compensator output serial vector.

\section{MODEL PARAMETERS' LMS IDENTIFICATION}

The adaptive coefficients $\omega$ are identified blindly by applying the LMS criterion to minimize the total energy of the distorted signals:

$$
\min _{\omega} J(\omega)=\min _{\omega}\left\{\left[S_{f}(\omega)\right]^{T} S_{f}(\omega)\right\}=\min _{\omega} \sum_{k=1}^{K}\left[s_{f}(k, \omega)\right]^{2}
$$

To obtain the solution for the nonlinear compensator coefficients, we could use the Gauss-Newton least squares method. In the case of cost function (4), the Gauss-Newton formula can be described as:

$$
\omega(i)=\omega(i-1)-\left\{Q^{T}[\omega(i-1)] Q[\omega(i-1)]\right\}^{-1} Q^{T}[\omega(i-1)] S_{f}[\omega(i-1)]
$$

$$
Q(\omega)=\left[\begin{array}{cccc}
\frac{\partial s_{f}(1, \omega)}{\partial \omega_{1}} & \frac{\partial s_{f}(1, \omega)}{\partial \omega_{2}} & \ldots & \frac{\partial s_{f}(1, \omega)}{\partial \omega_{N}} \\
\frac{\partial s_{f}(2, \omega)}{\partial \omega_{1}} & \frac{\partial s_{f}(2, \omega)}{\partial \omega_{2}} & \ldots & \frac{\partial s_{f}(2, \omega)}{\partial \omega_{N}} \\
\ldots & \ldots & \ldots & \ldots \\
\frac{\partial s_{f}(K, \omega)}{\partial \omega_{1}} & \frac{\partial s_{f}(K, \omega)}{\partial \omega_{2}} & \ldots & \frac{\partial s_{f}(K, \omega)}{\partial \omega_{N}}
\end{array}\right]
$$

Where, $i$ is the iteration number, $J(\omega)$ is the $K \times N$ Matrix.

Define $\quad v(k)=\left[y^{2}(k) \quad y(k) y(k-1) \ldots y^{2}\left(k-N_{d}+1\right) \quad y^{3}(k)\right.$ $\left.y^{2}(k) y(k-1) \ldots y^{D}\left(k-N_{d}+1\right)\right]^{T}$ as the polynomial of the memory nonlinearities, we can firstly rewrite (1) as

$$
s(k)=y(k)-v^{T}(k) \omega
$$

Then $S(k)$ can be expressed as

$$
\begin{gathered}
S(k)=Y(k)-V^{T}(k) \omega \\
Y(k)=\left[\begin{array}{llll}
y(k-B+1) & y(k-B+2) & y(k-498) \ldots y(k-1) & y(k)
\end{array}\right]^{T} \\
V(k)=\left[\begin{array}{lll}
v(k-B+1) & v(k-B+2) \ldots v(k-1) & v(k)
\end{array}\right]
\end{gathered}
$$

Where, $Y(k)$ is the $B \times 1$ vector, $V(k)$ is the $N \times B$ Matrix.

From (3) and (6), we can describe the $k$ th distortion signal and the total distortion energy of the $(i-1)$ th iteration as:

$$
s_{f}(k, \omega(i-1))=G^{T}\left[Y(k)-V^{T}(k) \omega(i-1)\right]
$$

Assumed that $k_{b}(i)$ and $k_{e}(i)$ denote the beginning and ending of the data window of the $i$ th iteration, respectively. We obtain the iteration formula of Volterra model parameters by expanding (5) according to the Gauss-Newton least squares method:

$$
\begin{aligned}
\omega(i)= & \omega(i-1)-\left[\sum_{k=1}^{K} V(k) G G^{T} V^{T}(k)\right]^{-1} \times \\
& \left\{\left[\sum_{k=1}^{K} V(k) G G^{T} V^{T}(k)\right] \omega(i-1)-\sum_{k=1}^{K} V(k) G G^{T} Y(k)\right\}
\end{aligned}
$$

Assume that $\Gamma$ is the constant $N \times N$ iteration step length matrix for updating the Volterra filter's coefficient vector. The LMS estimate of the nonlinear compensator coefficients are obtained as

$$
\begin{gathered}
\omega(i)=\omega(i-1)-\Gamma \times \\
\left\{\sum_{k=k_{b}(i-1)}^{k_{e}(i-1)} V(k) G G^{T} V^{T}(k) \omega(i-1)-\sum_{k=k_{b}(i-1)}^{k_{e}(i-1)} V(k) G G^{T} Y(k)\right\} \\
\Gamma=\left[\begin{array}{ccccc}
\Lambda_{2} & 0 & \ldots & 0 & 0 \\
\ldots & \ldots & \ldots & \ldots & \ldots \\
0 & \ldots & \Lambda_{d} & \ldots & 0 \\
\ldots & \ldots & \ldots & \ldots & \ldots \\
0 & 0 & \ldots & 0 & \Lambda_{D}
\end{array}\right], \\
\Lambda_{d}=\left[\begin{array}{ccccc}
\mu_{d} & 0 & \ldots & 0 & 0 \\
\ldots & \ldots & \ldots & \ldots & \ldots \\
0 & \ldots & \mu_{d} & \ldots & 0 \\
\ldots & \ldots & \ldots & \ldots & \ldots \\
0 & 0 & \ldots & 0 & \mu_{d}
\end{array}\right]
\end{gathered}
$$

Where, $\Lambda_{d}$ is the iteration step length matrix for updating the $d$ th Volterra filter's coefficient vector, $k_{b}(i)$ and $k_{e}(i)$ are the beginning and ending of the data window of the $i$ th iteration, respectively.

The computational complexity of the LMS estimate is $O(B \cdot M \cdot N)$. Table I shows the synthesis result comparison with three different parameter settings of $B, M$ and $N$ based on the LMS algorithms. Altera Quartus II was used to synthesize the design targeted towards the Stratix IV E FPGA. The computing capability of all the FPGA types in the Stratix IV E series could satisfy the real-time compensation requirement under the system operating frequency of $100 \mathrm{MHz}$. 
The change of nonlinearities could be reflected precisely if the sample length $M$ is big enough. Similarly, the total energy of the distortions could be calculated accurately if the order of the FIR bandstop filter $B$ is big enough. However, large $B, M$ and $N$ result in increased computational complexity [6] and large data length requirements for estimation purposes. In practice, the selection of appropriate $B, M$ and $N$ involves a tradeoff between good nonlinear compensation performance and low computational complexity. We set the parameters of the blind identification and compensation system implemented in FPGA as follows through the analysis and simulation on the required order and memory depth of the Volterra model.

- Sample length of the compensated output $M=1024$

- Order of the FIR bandstop filter $B=501$

- Highest order of the discrete Volterra Model $D=3$

TABLE I. SYNTHESIS RESULTS BASED ON LMS ALGORITHM

\begin{tabular}{|l|l|l|l|}
\hline & $B=201$ & $B=501$ & $B=1001$ \\
& $M=512$ & $M=1024$ & $M=2048$ \\
& $N=20$ & $N=31$ & $N=42$ \\
\hline ALUTs & 8693 & 15103 & 32127 \\
\hline Registers & 9828 & 18515 & 39232 \\
\hline BlockRAM(bit) & $2.76 \mathrm{M}$ & $3.19 \mathrm{M}$ & $4.24 \mathrm{M}$ \\
\hline 18-bit DSP & 183 & 371 & 749 \\
\hline
\end{tabular}

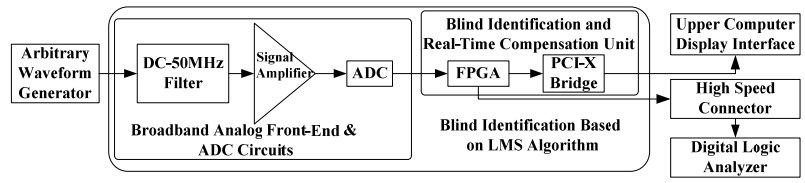

Figure 2. Experimental Verification Platform for the Blind Identification and Compensation Algorithm

- Memory depth of the 2nd order Volterra kernel $\mathrm{N}_{2}=6$

- Memory depth of the 3rd order Volterra kernel $N_{3}=3$

\section{EXPERIMENTAL RESULTS}

\section{A. Adaptive Iteration of the Objective $J(\omega)$}

Fig. 2 shows the experimental verification platform for the blind identification and compensation algorithm. Input signals were generated by the arbitrary waveform generator (AWG): PXI5422, produced by National Instrument. The broadband analog front-end and ADC circuits employed a cascade structure. The proposed blind identification and post-compensation technique was implemented in FPGA. The compensated output was transferred to the upper computer display interface through the PCI-X interface. The display interface was implemented in Microsoft Visual C++ 6.0. The intermediate data of the compensation process was transmitted to the digital logic analyzer through the high speed connector. Table II shows the performance of the component parts of the experimental verification platform for the blind identification and compensation algorithm.
Table III shows the fix point implementation design of the key parameters for the experimental verification.

The excitation input was an equal-amplitude multi-tone signal. The frequencies were 12.1 MHz, 16.2 MHz, 19.7 $\mathrm{MHz}$ and $21.9 \mathrm{MHz}$. The amplitude was $-1 \mathrm{dBFS}$ after amplifying. FS stands for full scale amplitude of ADC. We transferred the value of the objective to the digital logic analyzer through the high speed connector while the receiver system was running.

TABLE II. COMPONENT PERFORMANCE OF EXPERIMENTAL VERIFICATION PLATFORM

\begin{tabular}{|c|c|}
\hline & Performance \\
\hline AWG & $\begin{array}{l}\text { SFDR is } 79 \text { dBFS. Actual Measured Noise floor is }-110 \\
\mathrm{~dB}\end{array}$ \\
\hline Filter & Pass band is DC-50MHz. \\
\hline $\begin{array}{l}\text { Signal } \\
\text { Amplifier }\end{array}$ & Amplifier gain is $10.7 \mathrm{~dB} . \mathrm{P}_{-1}$ is $11.4 \mathrm{dBm}$ \\
\hline $\mathrm{ADC}$ & $\begin{array}{l}\text { Quantization bits are 16. Sampling rate is 100MSPS. } \\
\mathrm{V}_{\mathrm{p}-\mathrm{p}} \text { is } 2 \mathrm{~V} \text {. Two-tone SFDR is } 95 \mathrm{dBFS} \text {. } \\
\text { Operation mode is low pass sampling. }\end{array}$ \\
\hline FPGA & $\begin{array}{l}\text { Stratix IV E, speed grade is }-3 \text {. } \\
17.133 \text { Mbits BlockRAM, } 1288 \text { 18-bit DSP. }\end{array}$ \\
\hline $\begin{array}{l}\text { PCI-X } \\
\text { Bridge }\end{array}$ & $\begin{array}{l}\text { PCI6000 series produced by PLX. } \\
32 \text { bit bus width, } 100 \mathrm{MHz} \text { Operating Frequency }\end{array}$ \\
\hline
\end{tabular}

TABLE III. FIX POINT IMPLEMENTATION DESIGN OF KEY PARAMETERS FOR EXPERIMENTAL VERIFICATION

\begin{tabular}{|l|l|l|}
\hline & $\begin{array}{l}\text { Fix Point Data Width } \\
\text { (Including Sign Bit) }\end{array}$ & $\begin{array}{l}\text { Decimal } \\
\text { Width }\end{array}$ \\
\hline Input Data $y(k)$ & 16 bits & 19 bits \\
\hline $\begin{array}{l}\text { Memory Nonlinear } \\
\text { Polynomial } v(k)\end{array}$ & 23 bits & 30 bits \\
\hline Filter Coefficient $G$ & 16 bits & 15 bits \\
\hline $\begin{array}{l}\text { Compensation } \\
\text { Coefficient } \omega\end{array}$ & 32 bits & 34 bits \\
\hline $\begin{array}{l}\text { Compensated } \\
\text { Output Data } s(k)\end{array}$ & 16 bits & 19 bits \\
\hline
\end{tabular}

The floating point objective value was recovered from the fix point objective value according to the fix point implementation design shown in Table III. Fig. 3 shows the adaptive iteration of the objective $J(\omega)$. The LMS algorithm converged in about 57 times iterations.

\section{B. Performance of Blind Identification Algorithm}

Firstly, the excitation input was the equal-amplitude multi-tone signal mentioned in Section IV-A. The objective $J(\omega)$ reached its minimum value once it converged in several times' iterations. Fig. 4 shows the power spectrum comparison based on the LMS algorithm. The power spectrum was outputted to the display interface mentioned in Fig. 2. The sampling points number of the power spectrum was 131072 . The nonlinear distortion blind identification and compensation technique based on the LMS algorithm increased the SFDR of the system from 63.91 dBFS to 80.42 dBFS ignoring the low frequency disturbance. That was about $15 \mathrm{~dB}$ 's improvement.

Secondly, The excitation input was two 16-QAM modulation signals. The average power difference between 
the two signals was $50 \mathrm{~dB}$. The carrier frequency of the big signal was $17 \mathrm{MHz}$. The bandwidth of the big signal was 2 $\mathrm{MHz}$. The carrier frequency of the small signal was $20 \mathrm{MHz}$. The bandwidth of the small signal was $1 \mathrm{MHz}$. The sampling points number of the power spectrum was 65536. As the power spectrum shown in Fig. 5, the small signal was submerged by the nonlinear distortion of the big signal caused by the nonlinearities of the receiver's front end before compensation. The constellation obtained through narrow band filtering, down-conversion and periodic decimation scattered as the constellation diagram shown in Fig. 5. Therefore, the accurate identification could not be completed by the receiver's back-end. The ability of the system to detect weak signals was depressed. We applied the proposed blind identification and compensation method based on the LMS algorithm. After compensation, only the power of the noise floor and the small signal remained in the objective $J(\omega)$. The spectral spreading caused by the memory nonlinearities of the receiver's front end was eliminated. The adjacent-channel interference was suppressed. As a result, the small modulated signal emerged. Moreover, the constellation focused as shown in Fig. 5. The ability of the receiver system to detect weak signals was enhanced.

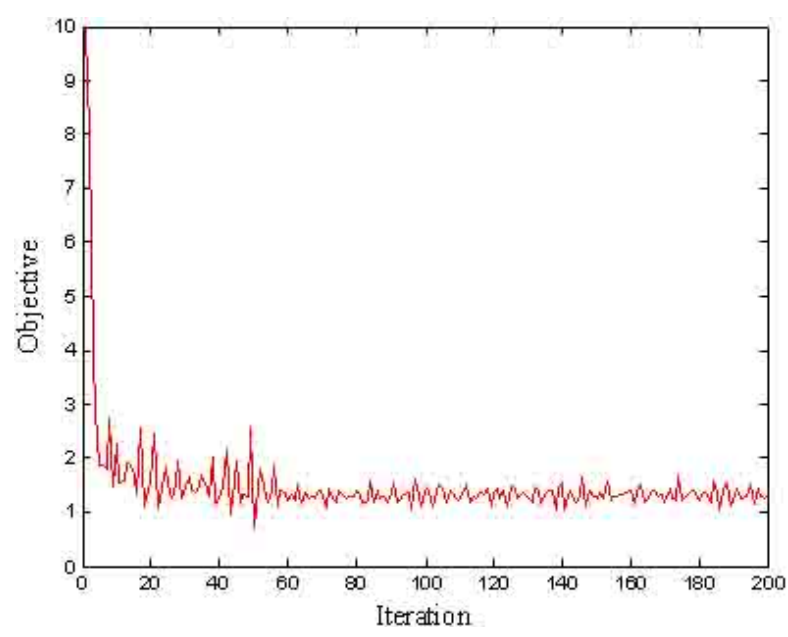

Figure 3. Adaptive iteration of objective $J(\omega)$

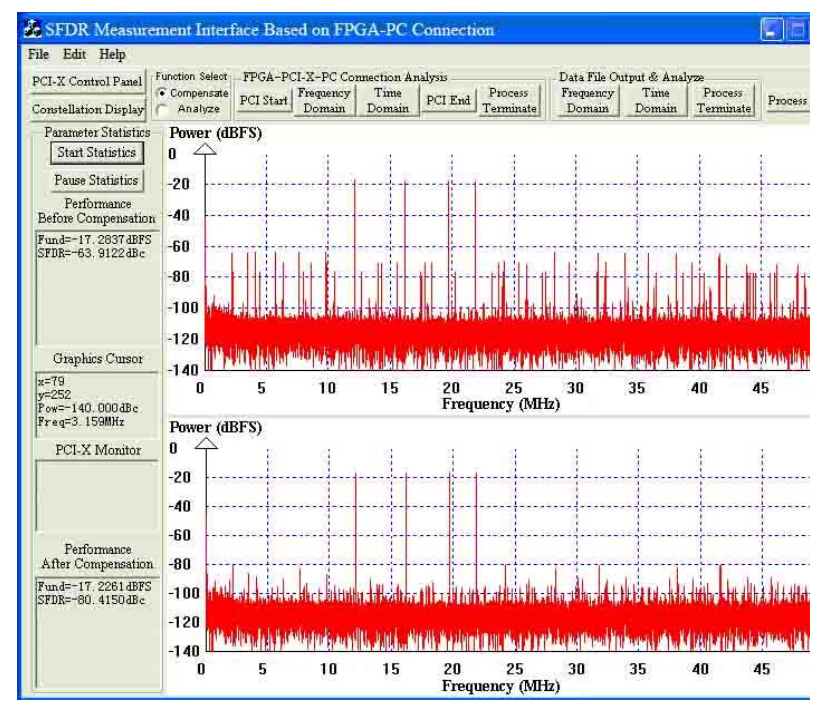

Figure 4. Power spectrum comparison between "before compensation" and "after compensation" based on LMS algorithm

\section{CONCLUSION}

We have designed and implemented the linearization tech- nique that aims to compensate the unknown memory nonlinear distortion by using a blind identification method based on the LMS algorithm. The technique can be considered as the natural evolution of the digital post calibration method [2],[3],[4],[5] toward the blind identification and the real-time compensation. The proposed solution removes the spectral spreading by estimating the coefficients of a Voterral model for the inverse nonlinearity.

\section{REFERENCES}

[1] Hu Xiao, Ma Hong, Peng Juan, Tian Chen, "State-of-the-Art in Volterra Series Modeling for ADC Nonlinearity", in the Second International Conference on Modeling and Simulation AMS2008,Kuala Lumpur, Malaysia, May,2008, pp. 1043-1047.

[2] N. Björsell, P. Suchanek, and P. Händel, "Measuring Volterra Kernels of Analog-to-Digital Converters Using a Stepped Three-Tone Scan", IEEE Trans. on Instrumentation and Measurement, Vol.57, No.4, 2008, pp.666-671

[3] Pavol Mikulik, Ján Saliga, "Volterra Filtering for Integrating ADC Error Correction, Based on an A Priori Error Model", IEEE Trans. on Instrumentation and Measurement, Vol.51, No.4, 2002, pp.870-875

[4] I.Cherif, S.Abid, Farhat Fnaiech, "Blind Nonlinear System Identification Under Gaussian And/Or I.I.D. Excitation Using Genetic Algorithms", IEEE International Conference on Signal Processing and Communications (ICSPC 2007), Vol.17, 2007

[5] Y. Chiu, C. W. Tsang, and B. Nikolic, "Least Mean Square Adaptive Digital Background Calibration of Pipelined Analog-to-Digital Converters", IEEE Trans. Circuits Syst. I, Vol.51, No.1, Jan 2004, pp. $38-46$

[6] J. Tsimbinos, K.V. Lever, "Computational Complexity of Volterra Based Nonlinear Compensators", IEE Electronics Letters, Vol.32, No.9, April 1996, pp.852-854 


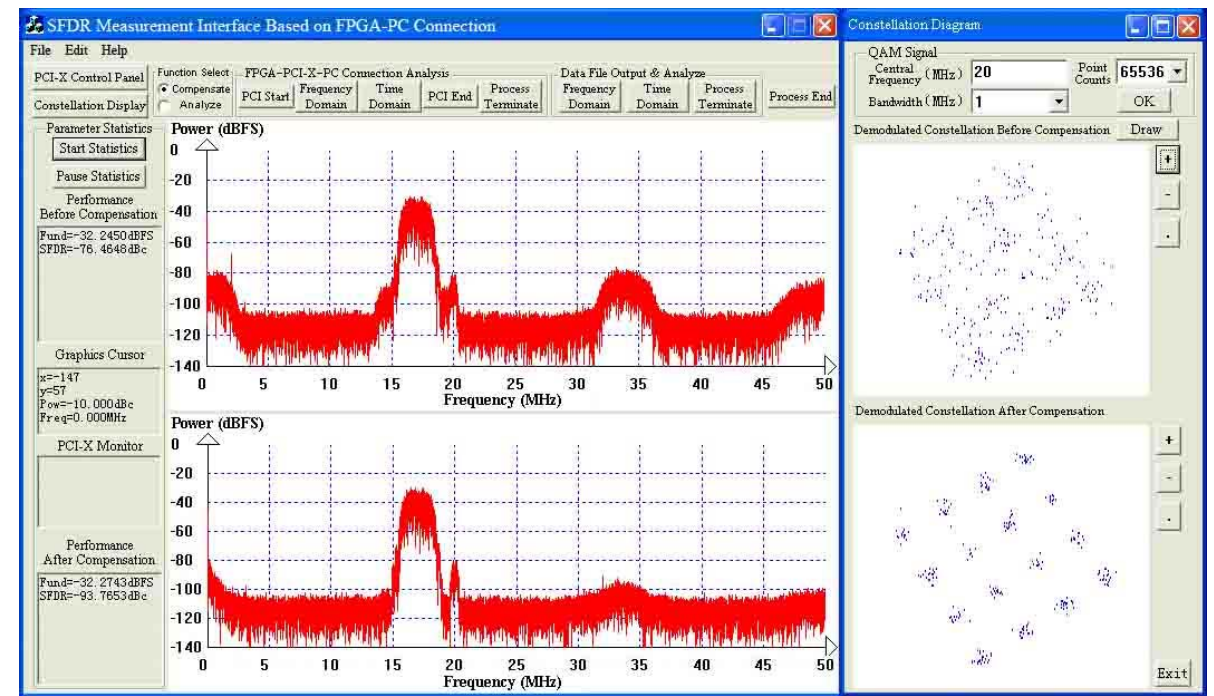

Figure 5. Power spectrum and constellation comparison between "before compensation" and "after compensation" 